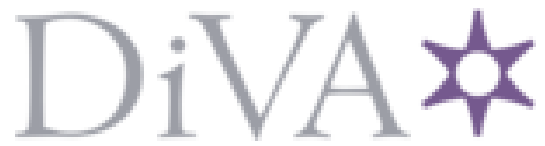

http://www.diva-portal.org

Preprint

This is the submitted version of a paper published in Journal of Food Engineering.

Citation for the original published paper (version of record):

Berta, M., Wiklund, J., Kotzé, R., Stading, M. (2016)

Correlation between in-line measurements of tomato ketchup shear viscosity and extensional viscosity

Journal of Food Engineering, 173: 8-14

https://doi.org/10.1016/j.jfoodeng.2015.10.028

Access to the published version may require subscription.

N.B. When citing this work, cite the original published paper.

Permanent link to this version:

http://urn.kb.se/resolve?urn=urn:nbn:se:ri:diva-427 


\title{
Correlation between in-line measurements of tomato ketchup shear viscosity and extensional viscosity
}

\author{
Marco Berta ${ }^{\text {a,* }}$, Johan Wiklund ${ }^{a}$, Reinhardt Kotzéa,c, Mats Stading ${ }^{\text {a, b }}$
}

${ }^{a}$ SP Food and Bioscience, PO Box 5401, SE-402 29 Gothenburg, Sweden

${ }^{b}$ Chalmers University of Technology, SE-412 96 Gothenburg, Sweden

${ }^{c b}$ FPRC - Flow Process \& Rheology Centre, Cape Peninsula University of Technology, PO Box 652, Cape Town 8000, South Africa

*Corresponding author: Tel.: +46(0)105166635, Fax: +46(0)313351363.

E-mail address: marco.berta@sp.se (M. Berta)

\begin{abstract}
The viscosity and shear thinning behaviour are essential characteristics of tomato ketchup. A real-time monitoring of those characteristics during processing is important to obtain a good quality of the final product and to reduce production waste. This work investigates the measurement of rheological in-line flow properties of tomato ketchup, using a real-time technique that combines ultrasound velocity profiling (UVP) and pressure difference (PD) assessment. In-line data were compared to those obtained off-line using a rotational viscometer. There was a poor correlation with the Bostwick measurement, whereas the flow curves calculated from flow velocimetry data were very similar to those measured off-line. The extensional viscosity of ketchup was determined through the measurement of Hyperbolic Contraction Flow; the curve followed a trend similar to that for the shear viscosity over the deformation rate investigated.
\end{abstract}




\section{Introduction}

The viscosity of tomato ketchup is a major quality component for consumer acceptance. A rigorous selection of ingredients and a continuous control and adjustment of the variables for processing are necessary to achieve a constant and desirable quality in the final product (Barringer et al., 1998; Bottiglieri et al., 1991; Jin Choi et al., 2006). Since small variations of processing conditions and raw materials affects the tomato ketchup flow behaviour and its final viscosity, several methods have been developed to monitor its flow and consistency during processing. The standard method for determining the tomato ketchup consistency is using a Bostwick consistometer (ASTM 2002). This is a quick and inexpensive measurement that is regularly carried out for samples taken during processing and for the final product. The relationship between the viscosity measurement by shear rheology and the Bostwick measurement has been studied by several authors (Barringer et al., 1998; Bottiglieri et al., 1991; Haley and Smith, 2003; McCarthy et al., 2008), but while rotational viscometry is more expensive and difficult to use for continuous quality monitoring, the Bostwick measurement presents other significant limitations. Bostwick measurement data are influenced by the operator skills, levelling, dryness/cleanness of the instrument, temperature of the sample, shape of the leading edge of the flow, and serum separation at the edge of the flow. In-line viscometry of tomato ketchup was developed to overcome these limitations, though at higher price. In-line ketchup viscosity measurements were performed by means of magnetic resonance (McCarthy and McCarthy, 2009), pressure differential (Barringer et al., 1998), in-line absorption photometry 
(Haley and Smith, 2003), and ultrasonic Doppler velocimetry (Jin Choi et al., 2006). Shear flow was characterized with these methods and it was assumed that this type of deformation was representative to the flow in the pipes during processing. An essential condition for this assumption is that the liquid sticks to the pipe wall and shearing is the main flow type, but it would not hold true if an abrupt restriction in pipe cross section occurs. In the latter case, as the fluid accelerates due to the rapid cross section change, extensional flow would become dominant and the deformation imposed by elongation would add to that imposed by shear (Cruz and Pinho, 2003; Debbaut and Crochet, 1988). Extensional deformation with the restriction of pipe section occurs often during tomato ketchup processing and when the final product is squirted out of a bottle. To have a complete description of its flow behaviour is then important to characterize the ketchup extensional viscosity together with the shear viscosity. Off-line extensional viscosity has been investigated generally for emulsions (Rao, 2014; Różańska, 2012; Różańska et al., 2013), but specific results for the tomato ketchup are lacking, mainly due to the experimental constraints and difficulties to obtain reliable data. In this study, off-line extensional viscosity measurements of commercial ketchup were carried out by hyperbolic contraction flow (Wikström and Bohlin 1999; Stading and Bohlin 2000). These results and off-line measurements of the shear viscosity were correlated to those obtained in-line with a rheometer that combines of Ultrasound Doppler Velocity Profiling (UVP) and the pressure difference (PD) technique (Claesson et al., 2013; Wiklund et al., 2013). This comparison aims to further the understanding of the tomato ketchup flow in pipes and to provide a base to evaluate its extensional viscosity from real time in-line measurements. 


\section{Materials and methods}

\subsection{Materials}

Commercial specimens of Heinz tomato ketchup were used for this study. All experiments were carried out at room temperature $\left(\approx 23^{\circ} \mathrm{C}\right)$.

\subsection{Rheological measurements}

\subsubsection{Continuous shear rheology}

The off-line apparent viscosity of the tomato ketchup was measured over a shear rate range of 0.1-100 $\mathrm{s}^{-1}$ using an Ares-G2 (TA instruments, Waters LLC, USA) strain controlled rotational rheometer equipped with a cone-plate geometry having a $40 \mathrm{~mm}$ diameter; $0.02 \mathrm{rad}$ cone angle, and $0.027 \mathrm{~mm}$ gap truncation. The yield stress was measured using a Reologica Stresstech stress controlled rotational rheometer equipped with a cone-plate geometry having a $30 \mathrm{~mm}$ diameter; $0.07 \mathrm{rad}$ cone angle, and $0.150 \mathrm{~mm}$ gap truncation.

\subsubsection{Hyperbolic contraction flow}

The tomato ketchup apparent extensional viscosity was measured using a Hyperbolic Contraction Flow rig(Wikström and Bohlin, 1999) mounted in an Instron 5542 Universal Testing Instrument (Instron Corporation, Canton, USA). Measurements were performed at room temperature using a die with inlet radius of $10 \mathrm{~mm}$ and outlet radius of $2 \mathrm{~mm}$ imposing a Hencky constant strain of 4.5. The extensional strain rates used were in the range $0.1 \mathrm{~s}^{-1}$, to $10 \mathrm{~s}^{-1}$, the data was evaluated as described previously(Binding, 1988; Stading and Bohlin, 2001). Experimental limitations such as

the instrument maximum speed and the formation of turbulent flow at high piston speed prevented the extensional viscosity measurement at higher rates. The transient extensional stress 
was monitored until a stable plateau value was reached from which the steady-state, transient extensional viscosity was calculated. The Power-law parameters acquired with the continuous shear measurements were used to calculate the extension rates, the Hencky strain and to compensate for the shear stress contribution to the total stress(Wikström and Bohlin, 1999). The calculated shear contribution was generally small $(<1 \%)$ and practically negligible.

\subsubsection{Pulsed ultrasound Doppler velocimetry and the enhanced tube viscometry method}

Ultrasound Doppler techniques were first introduced by Shigeo Satomura, a Japanese physicist, for practical medical diagnostics in the 1950s (Sigel, 1998). Pulsed ultrasound Doppler velocimetry and imaging techniques has since then been developed are now widely used for many academic and industrial applications(Takeda, 1995). Based on this technology, a novel inline fluid characterization system and method for complex industrial fluids and suspensions, Flow-Viz, has been developed over 15 years by SP and CPUT(Wiklund et al., 2014). Using the Flow-Viz method, the shear stress at the wall and the shear stress distribution inside the pipe is calculated from a pressure drop measurement over a fixed distance of pipe (Figure 1). The shear rate is estimated using a pulsed ultrasound sensor that allows true multi-point measurements of the liquid velocity distribution across the pipe diameter (Figure 1). The velocity gradient is measured from the pipe wall up to the position where it attains a constant velocity. This flat region of the velocity profile with constant velocity is commonly known as "plug flow region" (Chen et al., 1970; Dash et al., 1996; Slatter, 1997). The Flow-Viz can be regarded as a UVP+PD method since it combines Pulsed Ultrasound Velocimetry (UVP/PUV) with Pressure Difference measurements (PD) (Kotze et al., 2012; Wiklund et al., 2002; Wiklund et al., 2014; Wiklund et al., 2007). This method is non-invasive since it does not affect the current flow and does not give any additional pressure drop(Kotze et al., 2014). Since the measurements take only few seconds, 
the instrument software provides in real time, complete viscosity vs. shear rate distributions, i.e. flow curves and associated rheological model parameters such as the flow index, n, and consistency index, K. The fluid yield stress can also be estimated from the UVP data. It can be obtained either directly from the measured plug radius $\mathrm{R}^{*}$ in the velocity profile data or from a model fitting procedure (Equations 1-3). Figure 1 shows a schematic illustration of direct yield stress estimation from the plug velocity profile and the corresponding rheogram.

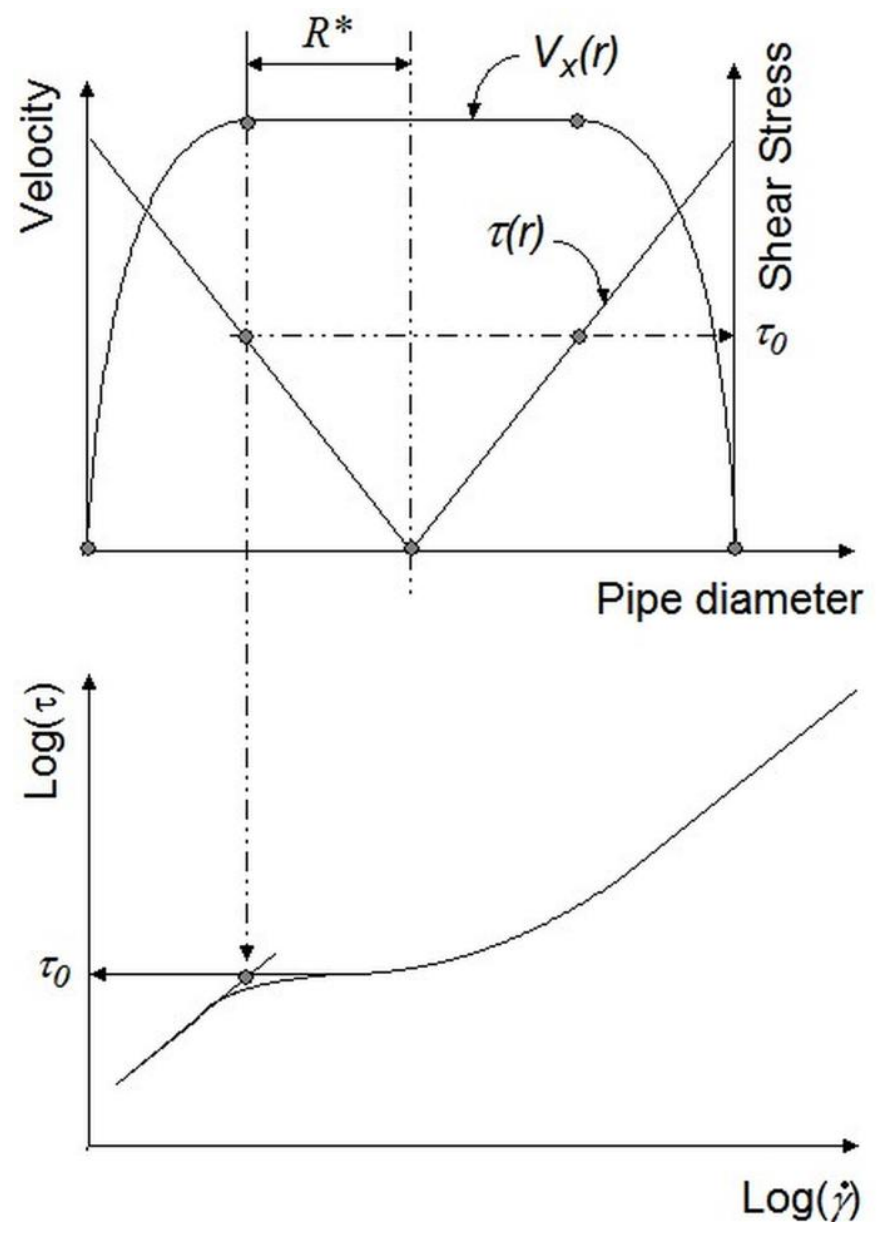

Fig. 1. Schematic illustration of yield stress estimation from the plug velocity profile, and the corresponding rheogram. 
The equation for the Herschel-Bulkley model is as follows:

$$
\tau=\tau_{y}+K(\dot{\gamma})^{n}
$$

Where consistency index $K$, the flow behaviour index $n$ and the yield stress $\tau_{y}$ are three curvefitting parameters. Equation 1 can be integrated to give the velocity $(v)$ profile across the pipe radius:

$$
v=\left(\frac{n}{(1+n)}\right)\left(\frac{\Delta P}{2 L K}\right)^{\frac{1}{n}}\left(\left(R-R^{*}\right)^{1+\frac{1}{n}}-\left(r-R^{*}\right)^{1+\frac{1}{n}}\right)
$$

where $R^{*}$ is the plug radius and is related to the fluid yield stress according to:

$$
R^{*}=\frac{2 L \tau_{y}}{\Delta P}
$$

The Herschel-Bulkley model can easily be modified to describe the power-law and Bingham plastic models (Slatter, 1997). Differences in yield stress measurements between the direct estimation method and from fitting measured data to theoretical models will depend on the quality of the velocity profile measurement and how it fluctuates within the given time interval. Yield stress values reported in this study were obtained directly from the plug radius measurement (Equation 3). To obtain in-line these data for the tomato ketchup, the Flow-Viz measuring system was connected to a flow loop (Figure 2), for which the flow rate could be varied from $5.3 \mathrm{l} / \mathrm{min}$ to $56.5 \mathrm{l} / \mathrm{min}$, corresponding to the industrial processing conditions. 


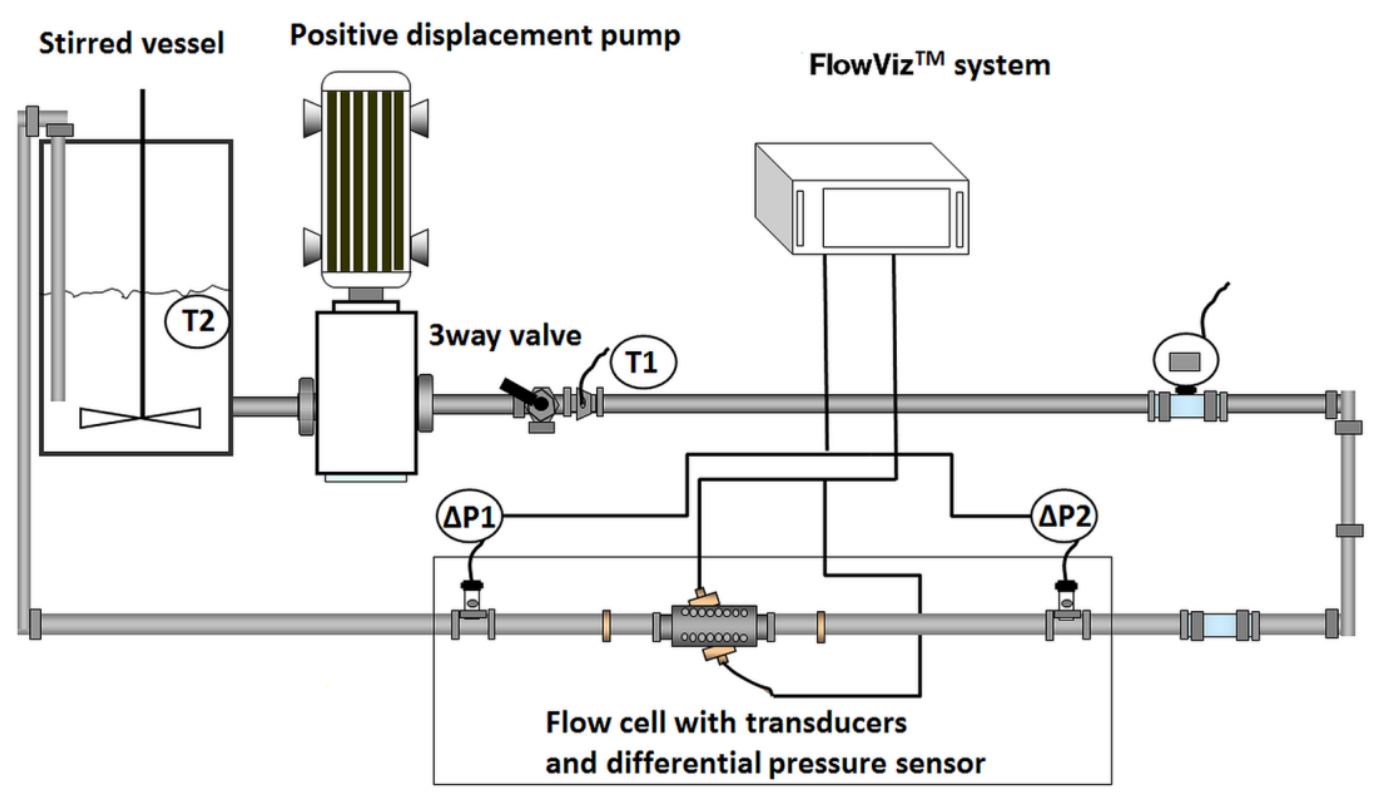

Fig. 2. Schematic illustration of the $51 \mathrm{~mm}$ flow loop (inner pipe diameter of $48.6 \mathrm{~mm}$ ), that was used for the measurements at SP Food and Bioscience, Gothenburg, Sweden. The sensor unit, highlighted with a rectangular box at the bottom, consists of a differential pressure sensor with remote seals and a novel ultrasound sensor unit.

The flow loop consisted of an agitated storage tank, a frequency-controlled positive displacement pump and a closed stainless steel piping circulation system including PT100 temperature sensors (Pentronic, Sweden), and an electromagnetic volumetric flow meter (Endress \& Houser, Sweden).

\subsubsection{Bostwick consistency measurements}

Flow lengths of the ketchup samples were measured using a standard Bostwick consistometer (CSC Scientific Company, Inc., Fairfax, VA) with sample compartment dimensions of 4.0 X 5.0 $X 5.0 \mathrm{~cm}$. After each sample was loaded into the compartment, the gate was opened, and the 
distance travelled by the leading edge of the concentrate was recorded after 30 seconds to the nearest $0.1 \mathrm{~cm}$. The tomato ketchup density $\rho$ was calculated by weighing out $200 \mathrm{ml}$ of product. With the Bostwick measurement, the yield stress is not straightforward to estimate as it is for example with shear rheology (Chhabra and Richardson, 2011). In this work we performed a $\mathrm{B}_{30}$ test, measuring the length of $3.5 \mathrm{~cm}$ of ketchup flowing after 30 seconds of Bostwick test. This aims to obtain a single-point value of the viscosity at one shear rate, according to the model proposed by McCarthy et al.(McCarthy and McCarthy, 2009). The equation proposed in (McCarthy and McCarthy, 2009) to determine the shear rate of the ketchup moving after 30s is

$$
\dot{\gamma}=\frac{\left(L^{\prime}\right)^{2}}{q t}
$$

Where L' is the sum of the length of the Bostwick sample compartment and the space covered by the flowing tomato ketchup after the time $t=30$ s. It is expressed in meters in the model calculation and for our test this value corresponds to $0.085 \mathrm{~m}$. The constant $\mathrm{q}$ is given by the volume/width of the sample compartment. The shear rate value for our test, according to Equation 4 , is $0.13 \mathrm{~s}^{-1}$. According to the model, the Bostwick measurement L' is related to the apparent viscosity and to the density of the fluid by the following formula:

$$
L^{\prime}=c\left(\frac{\eta}{\rho}\right)^{5}
$$

Where $\mathrm{c}$ is a constant that for the measurements in (McCarthy and McCarthy, 2009) corresponded to $0.0861, \eta$ the apparent viscosity at the shear rate given by Equation 4 , and $\rho$ the fluid density. 


\section{Results}

\subsection{Shear flow}

Instantaneous and radial velocity profiles were measured, non-invasively, for ketchup flow at several different flow rates. An example of a measured "raw" velocity profile or Doppler spectrum, from the wall to the center of the pipe is presented in Figure 3a. The corresponding normalized velocity profiles for three flow rates; 5.3, 19.9 and 56.6 L/min are presented in Figure 3b. The Doppler spectrum (Figure 3a) shows that a good signal-to-noise ratio was obtained.

The Flow-Viz system was found capable of measuring complete velocity profiles across the entire diameter of the pipe but only one half of the profile, from the wall to the center of the pipe is needed for rheological characterization. As it can be seen in Figure 3, the measured velocity profile showed the characteristic flattened profile shape of a non-Newtonian, shear-thinning fluid. It is not uncommon to see a non-zero velocity at the wall for this type of fluid and the non-zero velocity is normally interpreted as wall slip. However, due to the uneven distribution of scattering particles across the radius of the pipe, and in particular near the wall, it may be difficult to use the measured non-zero velocity at the wall as a direct measure of wall slip, even when the spatial resolution is high. Another factor is that the ultrasound sample volume overlaps the flowing fluid and wall interface. The flat region in the center of the pipe with constant velocity indicates the existence of a moving plug in which shearing of the sample takes place. The plug radius is directly related to the fluid yield stress, which can thus be determined directly from the measured data using Equation 3.

It is interesting to note that the internal structure was broken as the flow rate increased, which resulted in the Ketchup becoming more Newtonian (less shear-thinning) with increasing flow rate. This behavior is clearly seen in Figure $3 \mathrm{~b}$. The velocity profile at $5.31 / \mathrm{min}$ shows a decrease 
in magnitude between 0.01 and $0.015 \mathrm{~m}$. This is because a static echo was present which caused a loss of data in this region. Static echoes may occur due to various reasons: sensor mounting onto pipe and coupling material, type of fluid being tested, temperature changes and acoustic impedance differences. The velocity profile was corrected before being used for determining the rheology.

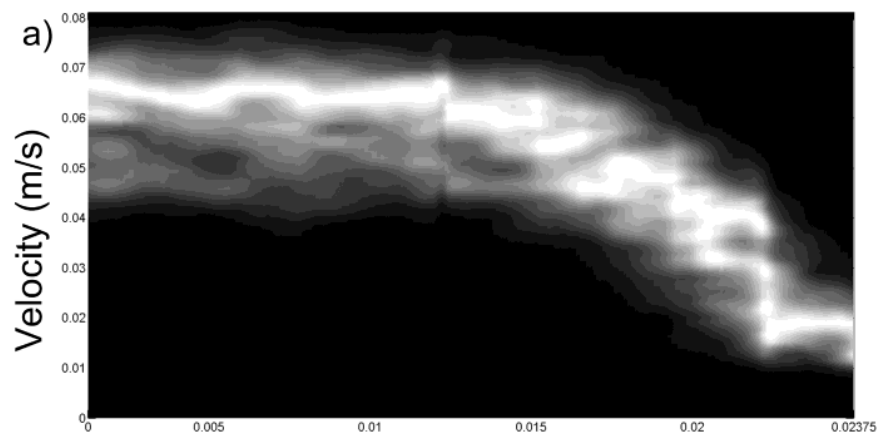

Pipe radius $(m)$

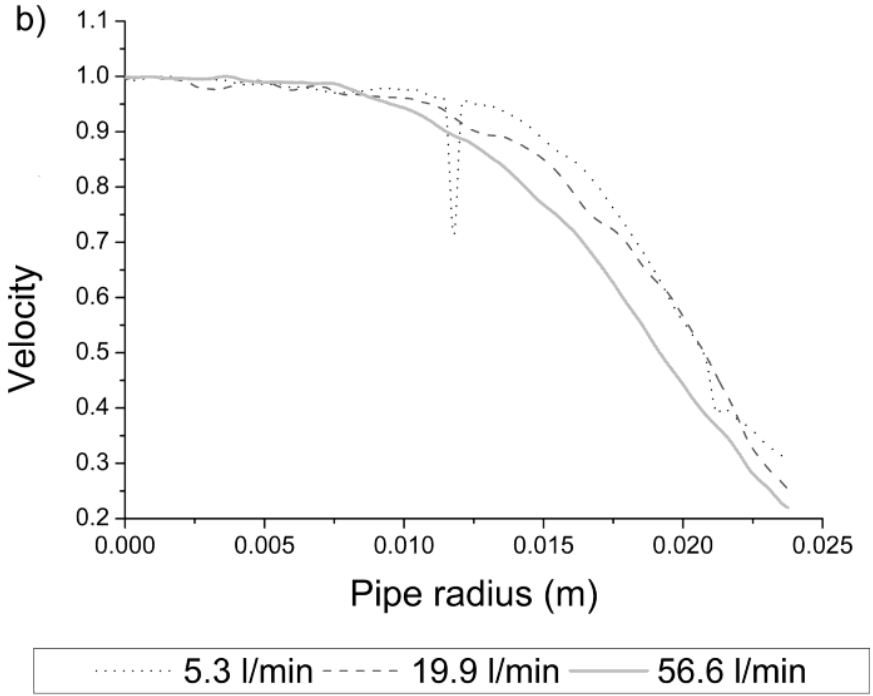

Fig. 3. a) Raw velocity profile for ketchup flow at $5.3 \mathrm{l} / \mathrm{min}$ and b) normalized curves extracted from the velocity data at 5.3, 19.9 and $56.61 / \mathrm{min}$.

The tomato ketchup flow curve measured off-line using a strain controlled Ares G2 rotational viscometer equipped with cone-plate geometry is compared to in-line measurements (Flow-Viz) 
in Figure 4. The material shear thinning flow curves could be fitted by the Ostwald de Waele power law model equation:

$$
\tau=K \dot{\gamma}^{n}
$$

where $\mathrm{K}$ and $\mathrm{n}$ are the consistency and flow behavior index, introduced for Equation 1.

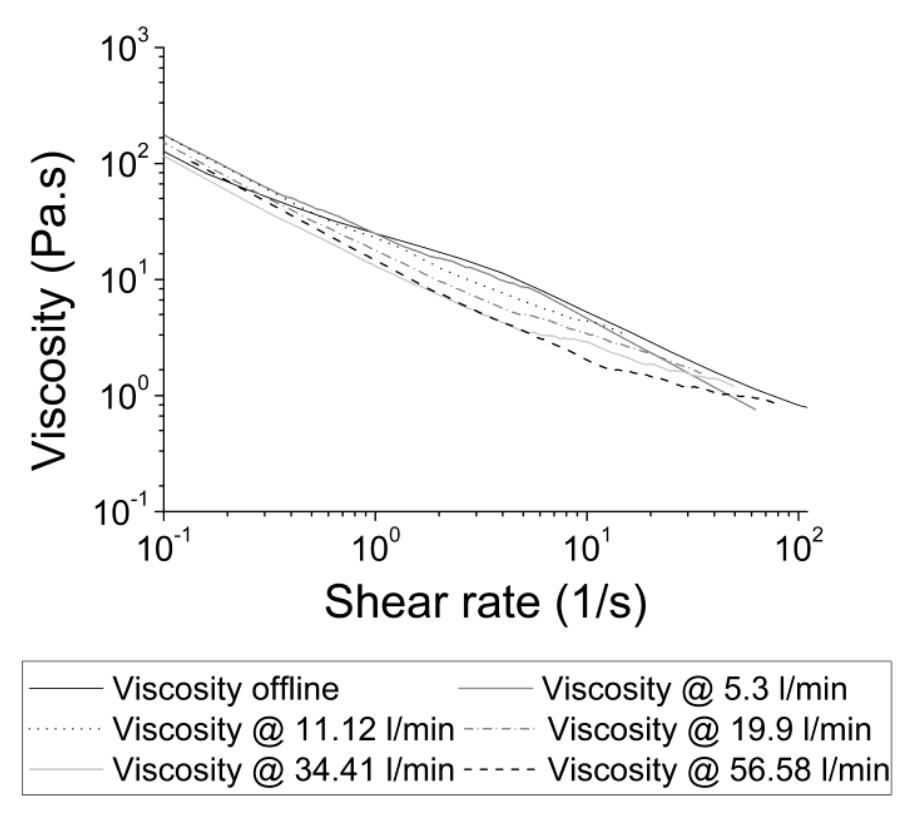

Fig. 4. Flow curves showing Off-line (rheometer) and In-line (Flow-Viz) measurements of the tomato ketchup apparent viscosity.

Since ketchup is commonly known as Bingham fluid, its yield stress was also estimated both from the off-line and in-line rheology measurements. For the in-line measurements the plug radius was measured and the yield stress estimated according to equation 3. A complementary off-line estimation of the ketchup yield stress was obtained using the stress controlled Stresstech rheometer. A stress ramp was performed as shown in Figure 5. 


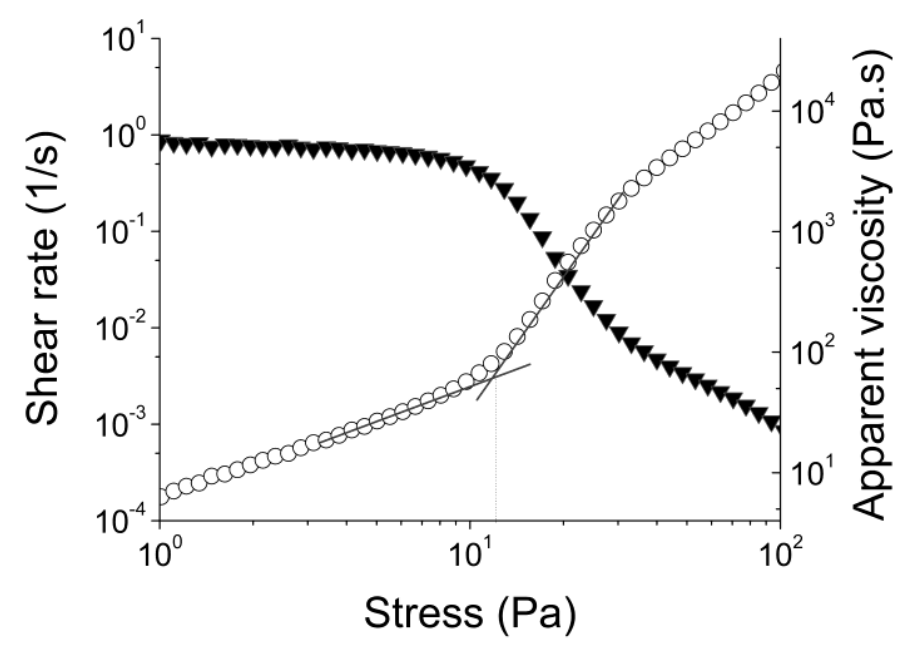

Fig. 5. Flow curves showing the $\bigcirc$ apparent viscosity and $\boldsymbol{\nabla}$ shear rate as a function of increasing stress. The drop in apparent viscosity corresponding to a kink of the shear rate curve indicates the change of flow regime. The stress at which this change occurs is the yield stress and it was calculated by the intersection of two linear fitting curves.

At low stress values the apparent viscosity was approximately constant and the shear rate increased slowly. As the stress increases a sudden drop of the apparent viscosity and a consequent increase of the shear rate occurred. These two flow regimes were fitted as shown in figure 5 and the yield stress $13.0 \pm 2.2 \mathrm{~Pa}$ was taken at the intersection of the two fitting lines. The values of yield stress obtained in-line are compared to that from off-line rheometry in Table 1 , and they are in the range of $14 \pm 1.7 \mathrm{~Pa}$. In Table 1 are reported also the values of $\mathrm{K}$ and $\mathrm{n}$ from the power law fitting of both off-line and in line flow curves.

\section{Table 1}

Power law parameters were obtained off-line for Heinz tomato ketchup using the ARES-G2 (strain control) while the yield stress was obtained using Stresstech (stress control) rheometer. 
The Flow-Viz system was used to estimate the same parameters in-line. Using the measured yield stress values, viscosity data have been fitted to the Herschel-Bulkley model (equation 1). The values of pressure difference over a fixed length $(\Delta \mathrm{P} / \mathrm{L})$ and plug radius (Rplug) from the FlowViz measurements are also reported.

\begin{tabular}{|c|c|c|c|c|c|}
\hline Method & $\mathrm{K}\left(\mathrm{Pa}_{\mathrm{s}} \mathrm{s}^{\mathrm{n}}\right)$ & $\mathrm{n}(-)$ & Yield stress $(\mathrm{Pa})$ & $\Delta \mathrm{P} / \mathrm{L}(\mathrm{Pa} / \mathrm{m})$ & Rplug (mm) \\
\hline ARES-G2*1 & 15.3 & 0.34 & & & \\
\hline Stresstech $* 2$ & & & 13.0 & & \\
\hline Flow-Viz 5.3 1/min & 12.8 & 0.35 & 15.4 & 3999.0 & 8.45 \\
\hline Flow-Viz 11.1 1/min & 9.5 & 0.4 & 16.2 & 4304.3 & 7.95 \\
\hline Flow-Viz 19.9 1/min & 4.6 & 0.65 & 12.7 & 4552.5 & 6.46 \\
\hline Flow-Viz $34.31 / \mathrm{min}$ & 2.1 & 0.81 & 12.7 & 5056.8 & 4.51 \\
\hline Flow-Viz 56.5 1/min & 0.8 & 0.98 & 13.2 & 5575.6 & 4.95 \\
\hline
\end{tabular}

The estimation of the apparent viscosity with the Bostwick method, using the parameters proposed in Equations 4 and 5, is $\approx 80$ Pa.s while that estimated using the Ares G2 rheometer is $\approx$ 100 Pa.s.

\subsection{Extensional flow}

The extensional flow curve is compared in figure 6 to that obtained using the ARES-G2 rotational shear rheometer. 


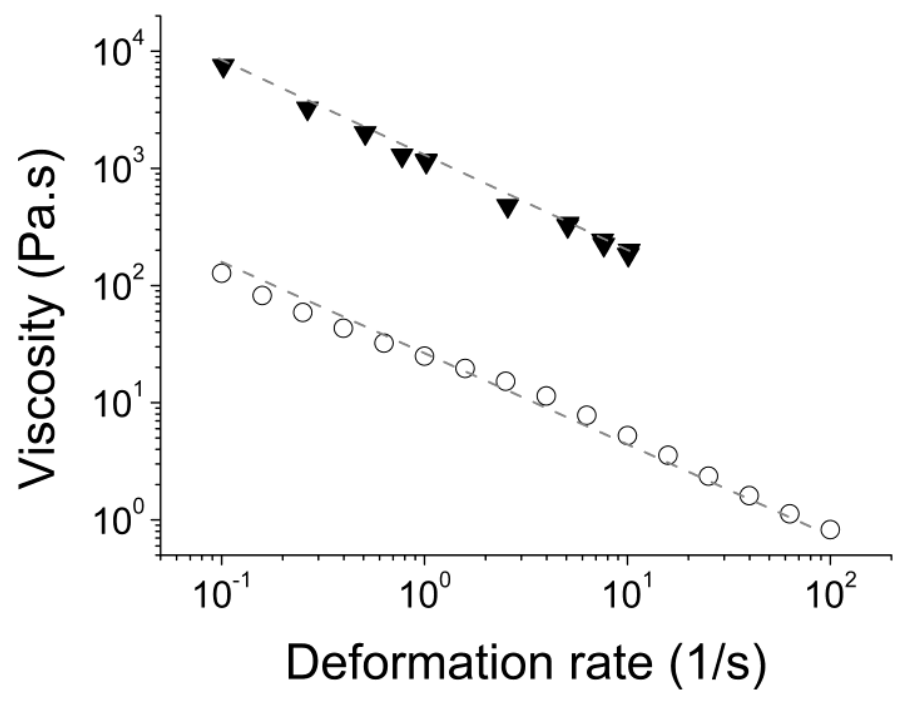

Fig. 6. Flow curves showing off-line measurements of the tomato ketchup apparent $\boldsymbol{\nabla}$ extensional viscosity $\eta_{\mathrm{e}}$ and $\circ$ shear viscosity $\eta$.

Despite the reduced range of deformation rate of the extensional flow curve compared to the shear flow curve, it is evident that both are well fitted by a power law (linear curves on a log-log scale) and that they are almost parallel. This is also shown by a similar $\mathrm{n}$ value of the power law fit, 0,21 for the extensional compared to 0.28 for the shear flow curve and implies an approximately constant Trouton ratio $T_{r}=\eta_{\mathrm{e}} / \eta$. This can be reasonably calculated as the ratio of the consistency indexes from the two fitting equations $\left(\mathrm{K}^{\prime} / \mathrm{K}\right)$ since in the Otswald-de Waele model it indicates the material viscosity range. Since the consistency index for the extensional flow curve fit is $\mathrm{K}^{\prime}=1158 \mathrm{~Pa}$, it follows that $\mathrm{T}_{\mathrm{r}} \approx \mathrm{K}^{\prime} / \mathrm{K} \approx 46$.

\section{Discussion}

The rheology data obtained with the Flow-Viz system correlate well with the offline data generated on the ARES-G2, when the flow rate is low (5.3 1/min). In Figure 4 it is shown that at 
higher pumping rates the flow curves deviate away from the off-line measurement. This is also suggested by a comparison of the power law parameters reported in table 1 . The consistency index $\mathrm{K}$ decreases while the flow behavior index $\mathrm{n}$ increases with increasing flow rates. The same was previously observed for vegetable sauce and seafood chowder flow and was explained by the increased shearing in the flow loop at increasing pumping rates(Wiklund and Stading, 2008). Despite these limitations, a visual inspection of the curves in Figure 4 shows that even the flow curve obtained in-line at a high rate $(56.6 \mathrm{l} / \mathrm{min})$ is close to the off-line flow curve at low shear rate. The yield stress values in table 1 show also similar values for off-line and in-line measurements, despite deviations for the latter are not directly related to the flow rate as for the power law coefficients.

The Bostwick consistometer allows only a punctual estimate of the apparent viscosity at one specific shear rate. According to the predictions using the model from McCarthy et al. (McCarthy and McCarthy, 2009), the estimate is of the same order of magnitude but still $20 \%$ far off that measured using the rotational rheometer ARES-G2. The accuracy may be improved by performing a calibration for the specific brand of tomato ketchup and calculating its own "c" coefficient to insert in Equation 5.

The extensional viscosity for this study was only measured off-line but it can also be performed in-line(Roëtynck, 2005). The extensional viscosity correlates well with the shear viscosity (Figure 6) for this specific fluid. The Trouton ratio was surprisingly larger than 3 (for Newtonian fluids) considering that ketchup is usually characterized as a dispersion. This may be due to a contribution from dissolved polysaccharides and interacting cell fragments that provides substantial elasticity. This may be also due to very low values of the calculated hyperbolic 
contraction flow shear stress $\tau_{\text {shear, }}$ which is subtracted to the measured stress $\tau_{\text {meas }}$ in order to obtain the value of stress produced by the extensional deformation $\tau_{\text {ext }}$ (Wikström and Bohlin 1999; Stading and Bohlin 2000). The extensional viscosity is calculated from the formula

$$
\eta_{e}=\frac{\tau_{\text {meas }}-\tau_{\text {shear }}}{\dot{\varepsilon}}=\frac{\tau_{\text {ext }}}{\dot{\varepsilon}}
$$

where $\dot{\varepsilon}$ is the extension rate. According to the model adopted, the shear stress in the hyperbolic die $\tau_{\text {shear }}$ is a function of the power law parameters $\mathrm{K}$ and $\mathrm{n}$ calculated from equation 6 and is expressed by:

$$
\tau_{\text {shear }}=\frac{4 H\left(3+\frac{1}{n}\right)^{n}\left(\frac{K}{\pi}\right)^{n} Q^{n}\left(\frac{1}{r_{0}^{3 n+1}}\right)^{n}}{3 n+3} X \frac{\left(\frac{r_{0}^{2}}{r_{1}^{2}}\right)^{\frac{3 n+3}{2}}-1}{\frac{r_{0}^{2}}{r_{1}^{2}}-1}
$$

Where $\mathrm{Q}$ is the volumetric flow rate through the die, function of the piston speed, $\mathrm{H}$ the die length, $r_{0}$ and $r_{1}$ the die entrance and exit diameter respectively. The shear stress predicted by equation 8 is plotted as a function of $\mathrm{K}$ and $\mathrm{n}$ in figure 7 , for a die with the dimensions of $\mathrm{r}_{0}=$ $0.01 \mathrm{~m}, \mathrm{r}_{1}=0.003 \mathrm{~m}, \mathrm{H}=0.015 \mathrm{~m}$ and flow rate of $1.5708 \mathrm{E}-07 \mathrm{~m}^{3} / \mathrm{s}$.

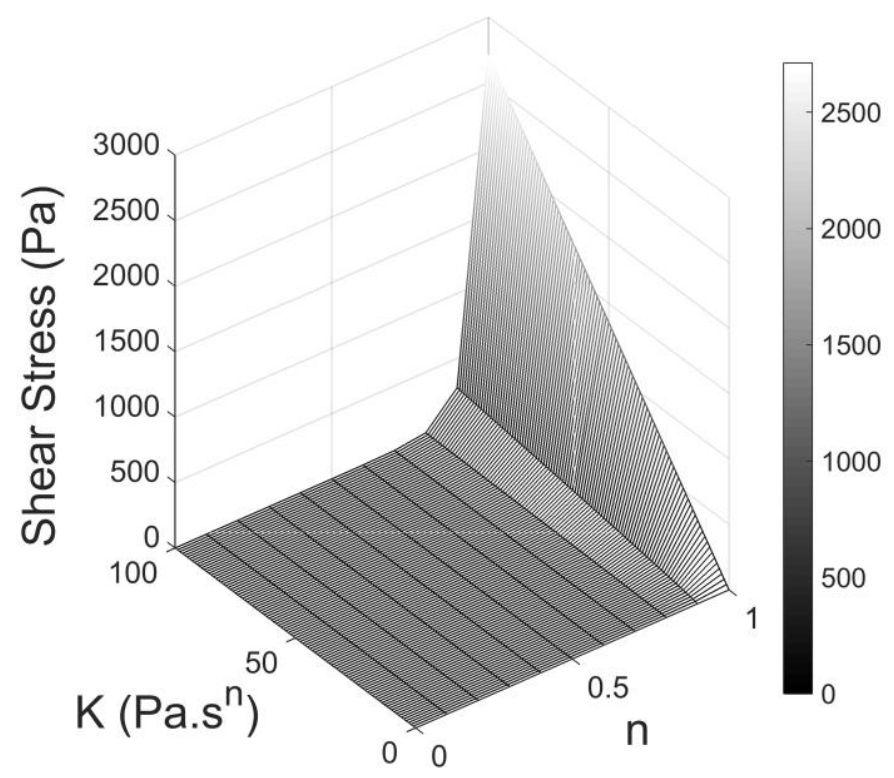


Fig. 7. 3D plot of the calculated shear stress in the Hyperbolic contraction flow die as a function of the power law coefficients K and n, according to the equation in (Wikström and Bohlin 1999; Stading and Bohlin 2000).

From the 3D plot in figure 7 it is evident that the shear stress estimated for the ketchup, with an $n$ value between 0.2 and 0.3 , is very low. A different correction and recalculation of the stress due to extensional deformation would require the establishment of a new model for the hyperbolic contraction flow. This is beyond the scope of the present publication and is suggested as further work. Since the slopes of extensional and shear flow curves were similar, the Trouton ratio was approximately constant within the range of deformation rate investigated $\left(0.1-10 \mathrm{~s}^{-1}\right)$.

\section{Conclusions}

This study found a correlation of the extensional viscosity flow curve to that of shear viscosity for the tomato ketchup, measured in-line by means of UVP+PD.

The shear data obtained the ultrasonic technique UVP+PD technique were very similar to shear data obtained off-line using a rotational shear viscometer. The tomato ketchup Trouton ratio was approximately constant within the range of deformation rates investigated $\left(0.1-10 \mathrm{~s}^{-1}\right)$, with a high value of 46 that may be related to the fluid elasticity. The Bostwick measurements combined with models proposed in the literature gave just a single apparent shear viscosity for one shear

rate, which roughly corresponded to the in-line and off-line measurements (80 Pa.s compared to $100 \mathrm{~Pa} . \mathrm{s})$. The direct in-line measurement of tomato ketchup extensional viscosity would be an interesting development of the same technique, and is planned as further work. 


\section{References}

Barringer, S.A., Azam, A.T.M.S., Heskitt, B., Sastry, S., (1998). On-line prediction of Bostwick consistency from pressure differential in pipe flow for ketchup and related tomato products. Journal of Food Processing and Preservation 22(3), 211-220.

Binding, D.M., (1988). An approximate analysis for contraction and converging flows. Journal of Non-Newtonian Fluid Mechanics 27(2), 173-189.

Bottiglieri, P., De Sio, F., Fasanaro, G., Mojoli, G., Impembo, M., Castaldo, D., (1991). Rheological characterization of ketchup. Journal of Food Quality 14(6), 497-512.

Chen, S.S., Fan, L.T., Hwang, C.L., (1970). Entrance region flow of the Bingham fluid in a circular pipe. AIChE Journal 16(2), 293-299.

Chhabra, R.P., Richardson, J.F., (2011). Non-Newtonian flow and applied rheology: engineering applications. Butterworth-Heinemann.

Claesson, J., Rasmuson, A., Wiklund, J., Wikström, T., (2013). Measurement and analysis of flow of concentrated fiber suspensions through a 2-D sudden expansion using UVP. AIChE Journal 59(3), 1012-1021.

Cruz, D.O.A., Pinho, F.T., (2003). Turbulent pipe flow predictions with a low Reynolds number $\mathrm{k}-\varepsilon$ model for drag reducing fluids. Journal of Non-Newtonian Fluid Mechanics 114(2-3), 109-148.

Dash, R.K., Mehta, K.N., Jayaraman, G., (1996). Casson fluid flow in a pipe filled with a homogeneous porous medium. International Journal of Engineering Science 34(10), 11451156. 
Debbaut, B., Crochet, M.J., (1988). Extensional effects in complex flows. Journal of NonNewtonian Fluid Mechanics 30(2-3), 169-184.

Haley, T.A., Smith, R.S., (2003). Evaluation of in-line absorption photometry to predict consistency of concentrated tomato products. LWT - Food Science and Technology 36(2), $159-164$.

Jin Choi, Y., Milczarek, R.R., Fleck, C.E., Casey Garvey, T., McCarthy, K.L., McCarthy, M.J., (2006). In-line monitoring of tomato concentrate physical properties during evaporation. Journal of Food Process Engineering 29(6), 615-632.

Kotze, R., Ricci, S., Wiklund, J., (2014). Performance tests of a new non-invasive sensor unit and ultrasound electronics, 9th International Symposium on Ultrasonic Doppler Methods for Fluid Mechanics and Fluid Engineering, Strasbourg, France, 105-108.

Kotze, R., Wiklund, J., Hadenwang, R., (2012). Optimization of the UVP+PD rheometric method for flow behavior monitoring of industrial fluid suspensions. Applied Rheology 22, 4276042761-42760-42711.

McCarthy, K.L., McCarthy, M.J., (2009). Relationship between In-line Viscosity and Bostwick Measurement during Ketchup Production. Journal of Food Science 74(6), E291-E297.

McCarthy, K.L., Sacher, R.F., Garvey, T.C., (2008). Relationship between rheological behavior and Bostwick measurement during manufacture of ketchup. Journal of Texture Studies 39(5), 480-495.

Rao, M.A., (2014). Measurement of Flow and Viscoelastic Properties, Rheology of Fluid, Semisolid, and Solid Foods. Springer US, 63-159.

Roëtynck, C.M., (2005). Comparison and utilisation of experimental extensional rheology techniques. Master of Science Thesis. Chalmers University of Technology. 
Różańska, S., (2012). Extensional Viscosity of w/o Emulsions. Procedia Engineering 42(0), $742-$ 752.

Różańska, S., Broniarz-Press, L., Różański, J., Mitkowski, P.T., Ochowiak, M., Woziwodzki, S., (2013). Extensional viscosity of o/w emulsion stabilized by polysaccharides measured on the opposed-nozzle device. Food Hydrocolloids 32(1), 130-142.

Sigel, B., (1998). A Brief History of Doppler Ultrasound in the Diagnosis of Peripheral Vascular Disease. Ultrasound in Medicine \& Biology 24(2), 169-176.

Slatter, P.T., (1997). The rheological characterisation of sludges. Water Science and Technology 36(11), 9-18.

Stading, M., Bohlin, L., (2001). Contraction flow measurements of extensional properties. Transactions Nordic Rheol. Soc. 8/9, 181-185.

Takeda, Y., (1995). Velocity profile measurement by ultrasonic doppler method. Experimental Thermal and Fluid Science 10(4), 444-453.

Wiklund, J., Haldenwang, R., Kotze, R., (2013). Fluid visualisation and characterisation system and method; a transducer. Google Patents.

Wiklund, J., Johansson, M., Shaik, J., Fischer, P., Windhab, E., Stading, M., Hermansson, A.-M., (2002). In-line ultrasound based rheometry of industrial and model suspensions flowing through pipes, Trans. Third International Symposium on Ultrasonic Doppler Methods for Fluid Engineering, EFPL, Lausanne, Switzerland, 69-76.

Wiklund, J., Kotze, R., Birkhofer, B., Ricci, S., Meacci, V., Hadenwang, R., Stading, M., (2014). M: Flow-VizTM - A fully integrated and commercial in-line fluid characterization system for industrial applications. Transactions Nordic Rheol. Soc. 22, 169-175. 
Wiklund, J., Shahram, I., Stading, M., (2007). Methodology for in-line rheology by ultrasound Doppler velocity profiling and pressure difference techniques. Chemical Engineering Science 62(16), 4277-4293.

Wikström, K., Bohlin, L., (1999). Extensional Flow Studies of Wheat Flour Dough. I.

Experimental Method for Measurements in Contraction Flow Geometry and Application to Flours Varying in Breadmaking Performance. Journal of Cereal Science 29(3), 217-226. Wiklund, J., Stading, M., (2008). Application of in-line ultrasound Doppler-based UVP-PD rheometry method to concentrated model and industrial suspensions. Flow Measurement and Instrumentation 19(3-4), 171-179. 